\title{
MicroRNA-27a Inhibits Cell Migration and Invasion of Fibroblast-Like Synoviocytes by Targeting Follistatin-Like Protein 1 in Rheumatoid Arthritis
}

\author{
Dong-liang Shi ${ }^{1}$, Gui-rong $\mathrm{Shi}^{2, *}$, Jing $\mathrm{Xie}^{1}, \mathrm{Xu}$-zhao $\mathrm{Du}^{1}$, and Hao Yang ${ }^{1}$
}

Fibroblast-like synoviocytes (FLS) with aberrant expression of microRNA (miRNA) are critical pathogenic regulators in rheumatoid arthritis (RA). Previous studies have found that overexpression or silencing of miRNA can contribute to the development of miRNA-based therapeutics in arthritis models. In this study, we explored the effects of miR-27a on cell migration and invasion in cultured FLS from RA patients. We found that miR-27a was markedly downregulated in the serum, synovial tissue, and FLS of RA patients. Meanwhile, the expression of follistatin-like protein 1 (FSTL1) was upregulated, which suggests that FSTL1 plays a key role in RA development. The results of a Transwell assay showed that miR-27a inhibited FLS migration and invasion. However, miR-27a inhibition promoted the migration and invasion of FLS. In addition, the downregulated expression of matrix metalloproteinases (MMP2, MMP9, and MMP13) and Rho family proteins (Rac1, Cdc42, and RhoA) was detected after treatment with miR-27a in RA-FLS by quantitative reverse transcription-PCR and western blot analysis. Then, a luciferase reporter assay validated that miR-27a targeted the 3-untranslated region (3'-UTR) of FSTL1. Moreover, miR-27a caused a significant decrease of FSTL1. In addition, the expression of TLR4 and NFKB was inhibited by miR-27a but increased by FSTL1 overexpression. In conclusion, we found that miR27a inhibited cell migration and invasion of RA-FLS by targeting FSTL1 and restraining the TLR4/NFKB pathway.

\section{INTRODUCTION}

Rheumatoid arthritis (RA) is a chronic joint inflammatory disease with abnormal synovial hyperplasia that leads to tissue destruction and functional disability (McInnes and Schett, 2011).

\footnotetext{
${ }^{1}$ No. 1 Department of Osteopathy, Henan Province Hospital of TCM, Zhengzhou, Henan, 450002, China, ${ }^{2}$ Shangqiu Medical College, Shangqiu, Henan, 456000, China

*Correspondence: shiguirongtcm@163.com
}

Received 20 April, 2016; revised 4 July, 2016; accepted 5 July, 2016; published online 8 August, 2016

Keywords: fibroblast-like synoviocyte, follistatin-like protein 1, microRNA, rheumatoid arthritis
Fibroblast-like synoviocytes (FLS) are key effector cells in the pathogenesis of RA (Bottini and Firestein, 2013). Previous reports have indicated that RA-FLS shares many properties with tumor cells: tumor-like proliferation, migration, and invasion, as well as increased resistance to apoptosis (Bottini and Firestein, 2013; Karouzakis et al., 2009). The invasion of FLS in RA is considered to be as aggressive as in tumor cells (Okamoto et al., 2007). FLS have inherent invasive qualities not observed in other fibroblasts. A previous study has demonstrated that intimal fibroblasts can invade articular cartilage even in the absence of immune cells because of these unusual properties (Fassbender and Simmling-Annefeld, 1983). Synoviocyte migration also was found to be partly responsible for spreading arthritic destruction to distant joints (Lefevre et al., 2009). Therefore, understanding the mechanism regulating the migration and invasion of FLS in RA may provide novel treatment strategies.

Follistatin-like protein-1 (FSTL1) was first identified as a transforming growth factor $\beta 1$-inducible protein, which is a secreted extracellular glycoprotein containing a follistatin-like and extracellular calcium-binding domain (Shibanuma et al., 1993). Previous studies revealed a substantial connection between FSTL1 levels and RA severity (Chaly et al., 2012). Some studies identified FSTL1 as a new pro-inflammatory mediator that contributes to RA by promoting the expression of tumor necrosis factor- $\alpha$, interleukin- $1 \beta$, interleukin- 6 , and interleukin- 8 , as well as by enhancing the interferon- $y$ signaling pathway in a mouse model (Chaly et al., 2012; Clutter et al., 2009). In this study, we observed aberrant levels and significant effects of FSTL1 in RA -FLS.

MiRNAs are endogenous, small (21-25 nucleotides), singlestranded, non-coding RNAs that mediate mRNA cleavage, translational repression, and mRNA destabilization (Luo et al., 2010). It has been reported that alterations in miRNA expression levels impact the expression of target genes in a 1.0-fold to 4-fold manner, which indicates that a fine-tuning mechanism regulates protein expression ( $\mathrm{O}^{\prime}$ Connell et al., 2012). The altered expression of circulating miRNAs in RA-FLS has been reported repeatedly (Gallo et al., 2014; Nakasa et al., 2011; Yang and Yang, 2015). In particular, previous reports have demonstrated that miR-27a has key roles in the migration and invasion of cancer cells ( $\mathrm{Li}$ et al., 2015b; Pan et al., 2014). A recent study showed that miR-27a was dysregulated in chondrocytes in osteoarthritis (Tardif et al., 2009). However, the role of miR-27a in RA has remained elusive. 
Matrix metalloproteinases (MMPs), a family of zinc-dependent endopeptidases, are the main proteases responsible for the invasion and degradation of basement membranes and extracellular matrix. MMP2, MMP9, and MMP13 are key members of the MMP family that can cleave gelatin and facilitate cell migration and invasion (Zhang et al., 2015). It has been demonstrated that herbal extracts inhibit human umbilical vein endothelial cell invasion and migration by regulating MMP2 and MMP9 (Ding et al., 2013; Pratheeshkumar and Kuttan, 2011). RA-FLS can erode cartilage by synthesizing and secreting MMPs (Abeles and Pillinger, 2006), thus representing a potential target for RA therapy. The Rho family of small GTPases forms a 20-member family within the Ras superfamily of GTPdependent enzymes that are activated by a variety of extracellular signals. RhoA, Cdc42, and Rac1, the most well-known Rho family members (Liang et al., 2015; Yuan et al., 2014), all have been reported to play important roles in the migration and invasion of various cells (Lv et al., 2015).

In our study, the expression of MMPs (MMP2, MMP9, MMP13) and Rho family members (RhoA, Cdc42, Rac1) may be associated with the cell migration and invasion of RA-FLS. Therefore, we analyzed aberrant expression of miRNA-27a and FSTL1 in RA patients and then focused on the effects of miR27a on RA-FLS migration and invasion. The mechanism of miR-27a-mediated migration and invasion-related protein expression was also determined in RA-FLS.

\section{MATERIALS AND METHODS}

\section{Preparation of human serum, synovial tissues, and} fibroblast-like synoviocytes

This study was performed according to the recommendations of the Declaration of Helsinki and approved by the Medical Ethical Committee of the Henan Province Hospital of TCM in Henan, China. Serum and synovial tissue samples were obtained from patients with RA (10 women and 8 men, 40-68 years old) who were undergoing synovectomy or joint replacement at the hospital. Eighteen healthy subjects (12 men and 6 women; age range, 38-65 years old) were used as normal controls. All patients provided their informed consents to participate in the study. RA was diagnosed according to the 2010 ACR/European League against Rheumatism classification criteria for RA (Aletaha et al., 2010). Synovial tissue samples were cut into small pieces and digested with $1 \mathrm{mg} / \mathrm{ml}$ collagenase for $3 \mathrm{~h}$ at $37^{\circ} \mathrm{C}$ to isolate synoviocytes. RA-FLS and human embryonic kidney 293 cells were cultured in DMEM/F12 with $10 \%$ FBS, 100 units/ml penicillin, and $100 \mu \mathrm{g} / \mathrm{ml}$ streptomycin at $37^{\circ} \mathrm{C}$ and $5 \% \mathrm{CO}_{2}$, as previously described (Lv et al., 2015). In our experiments, cells were used at passages from 3 to 6 , during which time they were a homogeneous population of cells.

\section{Enzyme-linked immunosorbent assay (ELISA)}

Serum FSTL1 levels were measured using a standard quantitative sandwich ELISA (eBioscience, Austria). All analyses and calibrations were performed in triplicate.

\section{Cell transfection of miR-27a mimic and inhibitor}

FLS were seeded into 24-well or 6-well plates $24 \mathrm{~h}$ prior to transfection. MiRNA control mimic, miR-27a mimic, miRNA control inhibitor, and miR-27a inhibitor (Applied Biosystems, USA) were transfected at a final concentration of $80 \mathrm{nM}$ with Lipofectamine 2000 (Invitrogen, USA). The cells were harvested at $24 \mathrm{~h}$ for RNA extraction and at $48 \mathrm{~h}$ for protein extraction.
Cell migration and invasion assays

The cell migration assay was performed with Transwell chambers with $8-\mu \mathrm{m}$ pores (Corning, USA). FLS $\left(1 \times 10^{5}\right.$ cells per chamber) were plated in the upper chambers in duplicate filters. DMEM containing 10\% FBS was added to the lower chamber as a chemoattractant. FLS were suspended in serum-free DMEM in the upper chambers. Forty-eight hours after transfection with the miR-27a mimic or miR-27a inhibitor, non-migrating cells were removed from the upper surface, and filters were stained with crystal violet. Migrated cells were counted in five representative microscopic fields (100× magnification).

Cell invasion ability was assessed using Matrigel invasion chambers (BD Biosciences, USA). FLS $\left(5 \times 10^{4}\right.$ cells per chamber) were seeded onto Matrigel Transwell chambers pre-coated with Matrigel (BD Biosciences). The lower chambers were filled with DMEM medium containing $10 \%$ FBS, and the FLS in the upper chambers were filled with serum-free DMEM. Forty-eight hours after transfection with the miR-27a mimic or miR-27a inhibitor, cells on the top membrane surface were removed. Cells that penetrated to the bottom were stained with crystal violet and counted, as previously described.

\section{Construction of adenoviral vector expressing FSTL1}

The wild-type FSTL1 cDNA sequence without the 3'UTR was subcloned into an adenovirus shuttle vector. After linearization, the shuttle vector was co-transformed into Escherichia coli with the adenoviral backbone plasmid pAdEasy-1 (Stratagene, USA). The resultant recombinant adenoviral DNA with FSTL1 cDNA was transfected into human embryonic kidney 293 cells to produce the recombinant adenoviral vector. An adenoviral vector expressing $\beta$-galactosidase (Ad- $\beta$ gal) was used as a control. Adenoviral vectors were purified by $\mathrm{CsCl}$ ultracentrifugation.

\section{Quantitative reverse transcription-PCR}

Total RNA was extracted from both synovial tissues and FLS with TRIzol reagent (Invitrogen). For mRNA quantification, 500 ng of RNA were used for the synthesis of CDNA with reverse transcriptase using the M-MLV First Strand Kit (Taraka, China), according to the manufacturer's instructions. One microliter of cDNA was used for real-time PCR with GoTaq qRT-PCR Master Mix (Promega, USA). For each sample, the relative mRNA level was normalized to $\beta$-actin expression. Table 1 lists the primers for qRT-PCR.

For miRNA quantification, the GoScript Reverse Transcription System Kit (Promega) was used with the stem loop primer. For each sample, the relative mRNA level was normalized by U6. The forward and reverse primers for miR-27a were $5^{\prime}$ UUCACAGUGGCUAAGUUCCGC-3' and 5'-GGAACUUAGCC ACUGUGAAUU-3'. Relative expression levels of miRNA or mRNA were analyzed using the Bio-Rad C1000 Thermal Cycler (Bio-Rad, USA).

\section{Western blot}

Total membranes and nuclear extracts were prepared as described previously, and protein concentrations were determined using the BCA assay (Pierce, USA). The dilution of primary antibodies was as follows: MMP2 (MA1-772, 1:500); MMP9 (MA5-14220, 1:500); MMP13 (MA5-14247, 1:350); FSTL1 (PA5-31113, 1:1500); Rac1 (PA1-091, 1:1000); Cdc42 (PA1-092X, 1:1000); RhoA (MA1-011, 1:1000); TLR4 (MA516216, 1:1000); and NF-KB (PA1-186, 1:1000). All primary antibodies were detected with rabbit polyclonal antibodies (Invitrogen). Each membrane was rinsed three times for 15 min and 
Table 1. RT-PCR primer sequences

\begin{tabular}{|c|c|}
\hline GENE & Primer sequences $\left(5^{\prime}-3^{\prime}\right)$ \\
\hline \multirow[t]{2}{*}{ FSTL1 } & F: CCAGAACTATGATAATGGAGACGCT \\
\hline & R: TAAGATGAACTATGAACCTCCTGCC \\
\hline \multirow[t]{2}{*}{ MMP2 } & F: CCGTCGCCCATCATCAAGTT \\
\hline & R: CTGTCTGGGGCAGTCCAAAG \\
\hline \multirow[t]{2}{*}{ MMP9 } & F: TCCTGGTGCTGGCTTGCTGC \\
\hline & R: CAATGTCAGCTTCGGGGCCG \\
\hline \multirow[t]{2}{*}{ MMP13 } & F: TGATGAAACCTGGACAAGCA \\
\hline & R: TCCTCGGAGACTGGTAATGG \\
\hline \multirow[t]{2}{*}{ Rac1 } & F: GTAAAACCTGCCTGCTCATC \\
\hline & R: GCTTCGTCAAACACTGTCTTG \\
\hline \multirow[t]{2}{*}{ Cdc42 } & F: GCAGGGCAAGAGGATTATGA \\
\hline & R: GGTCCCGACAAGCAAGAAA \\
\hline \multirow[t]{2}{*}{ RhoA } & F: CATCCCAGAAAAGTGGACTCCA \\
\hline & R: CCTTGTGTGCTCATCATTCCG \\
\hline \multirow[t]{2}{*}{ TLR4 } & F: CAGCTCTTGGTGGAAGTTGA \\
\hline & R: GCAAGAAGCATCAGGTGAAA \\
\hline \multirow[t]{2}{*}{ NF-kB p65 } & F: CTCTGCTTCCAGGTGACAGT \\
\hline & R: TCCTCTTTCTGCACCTTGTC \\
\hline \multirow[t]{2}{*}{$\beta$-actin } & F: GTGGGGCGCCCCAGGCACCA \\
\hline & R: CTCCTTAATGTCACGCACGATTTC \\
\hline
\end{tabular}

F, Forward sequences; $R$, Reverse sequences

incubated with the secondary horseradish peroxidase-linked antibodies. $\beta$-actin was used as the loading reference for data analysis.
Luciferase reporter assay

The human FSTL1 3'-UTR was amplified and cloned into the Xbal site of the pGL3-control vector (Invitrogen), downstream of the luciferase gene, to generate the pGL3-FSTL1-3'UTR plasmid. In addition, pGL3-FSTL1-3'UTR-mut plasmids were constructed using CDNA fragments containing corresponding mutated nucleotides for miR-27a as a control. For the luciferase reporter assay, FLS were co-transfected with $100 \mathrm{ng}$ of the luciferase reporter vectors and $20 \mathrm{pmol}$ of control mimic, miR27a mimic, control inhibitor, or miR-27a inhibitor using Lipofectamine 2000 (Invitrogen). The $\beta$-actin promoter Renilla luciferase reporter was used for normalization. After $48 \mathrm{~h}$, luciferase activity was analyzed using the Dual-Luciferase Assay System (Promega).

\section{Statistical analysis}

All data are expressed as the mean \pm SD of results derived from three independent experiments performed in triplicate. Statistical analysis was performed by Student's $t$-test and ANOVA. A difference was accepted as significant if $p<0.05$.

\section{RESULTS}

MiR-27a expression is downregulated and FSTL1 expression is upregulated in the serum, synovial tissue, and fibroblast-like synoviocytes of rheumatoid arthritis patients

The miR-27a expression and FSTL1 levels in the serum, synovial tissue, and FLS of RA patients and healthy controls were determined using qRT-PCR and Western blot analysis. It was found that FSTL1 expression in the serum, synovial tissue, and FLS was significantly elevated in RA patients, compared to healthy controls (Fig. 1A). A microRNA database was used to screen miRNA candidates targeted to FSTL1 (Edris, 2011). As
A
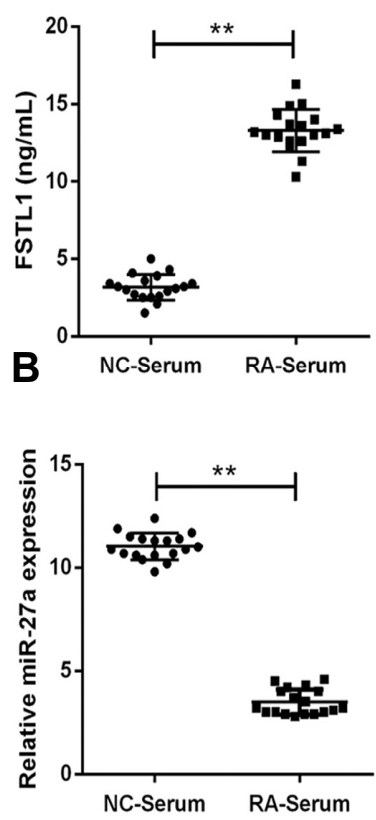
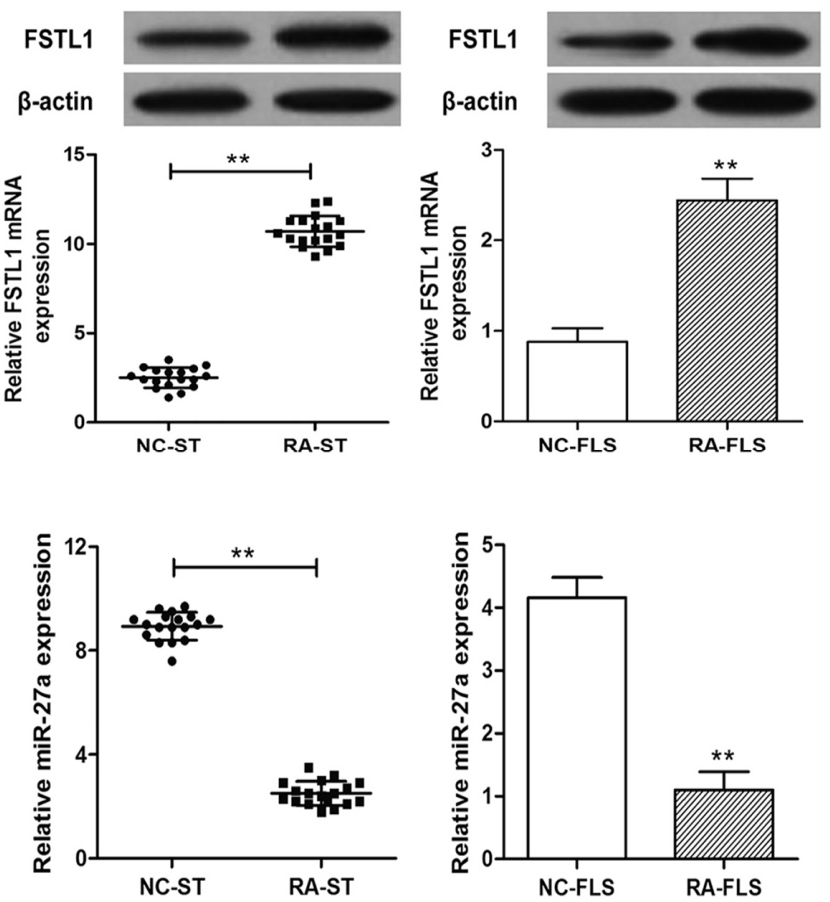

Fig. 1. Expression of miR-27a and FSTL1 in RA serum, synovial tissue, and FLS. (A) The serum expression of FSTL1 of RA patients was validated by ELISA, and its level in synovial tissue and FLS was validated by western blot and qRT-PCR. (B) The expression of miR-27a was validated in serum, synovial tissue, and FLS of RA patients by qRT-PCR. ${ }^{* *} p<$ 0.01 , versus control group. 


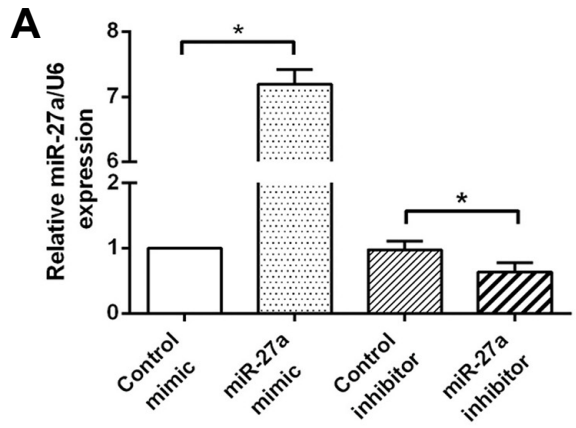

B
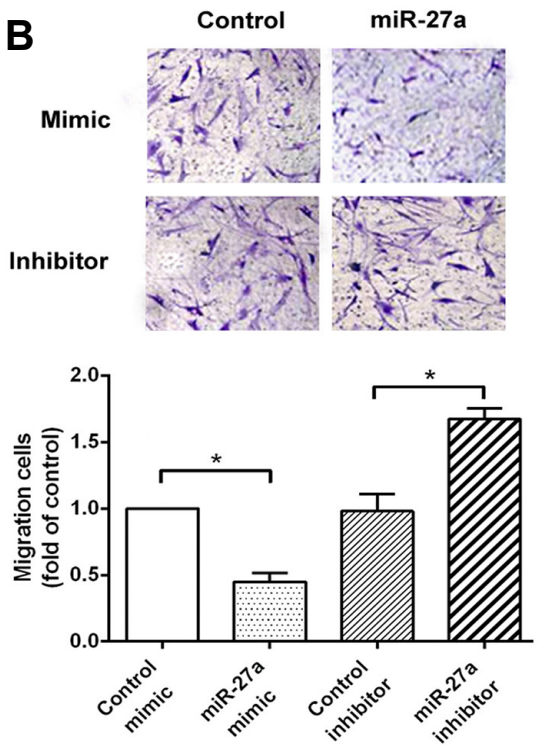

A

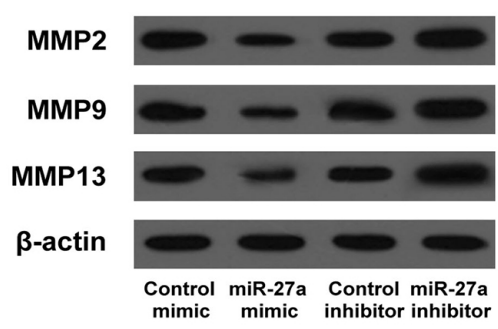

C

Rac1

Cdc42

RhoA

$\beta$-actin
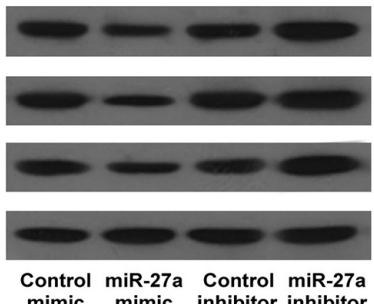

Control miR-27a Control miR-27a
mimic mimic inhibitor inhibitor

B

D

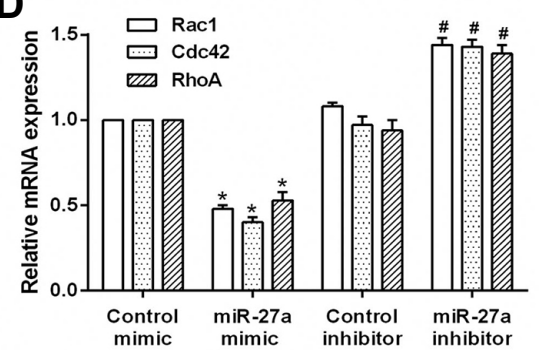

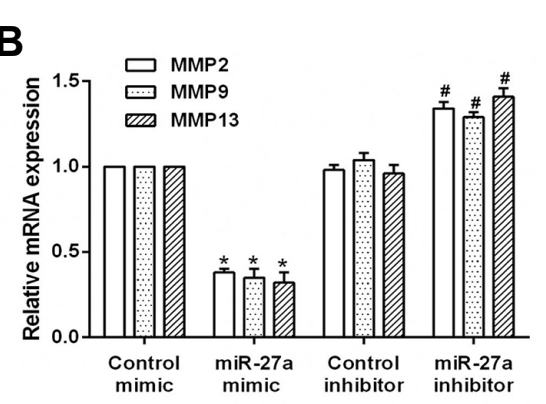

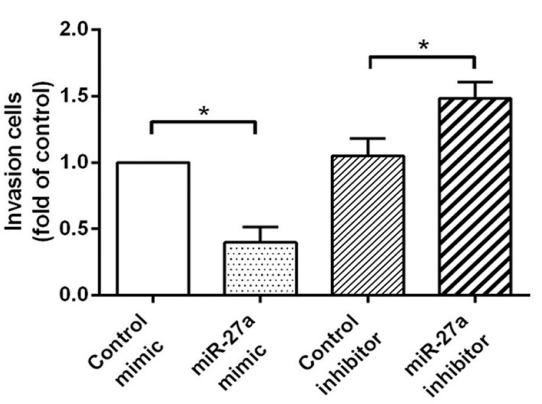

Fig. 2. Effects of miR-27a on the cell migration and invasion of RA-FLS. (A) The expression of miR-27a was detected by qRTPCR after the transfection of miR-27a or miR-27a inhibitor. (B) RA-FLS migration was measured using the Transwell system after transfection with the miR-27a mimic or miR27a inhibitor. (C) RA-FLS invasion was measured using the Transwell system after transfection with the miR-27a mimic or miR$27 a$ inhibitor. ${ }^{*} p<0.05$, versus control group.

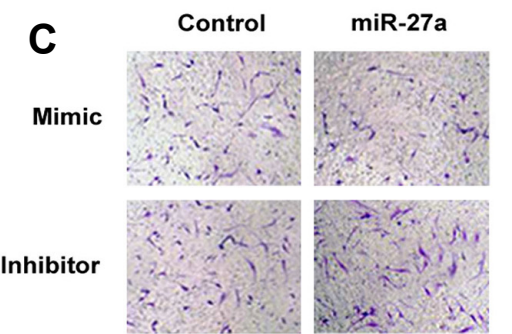

Fig. 3. Effects of miR-27a on the expression of migration and invasion-related proteins in RA-FLS. RA-FLS were transfected with miR-27a mimic or miR-27a inhibitor. (A) The protein and mRNA expression of MMP2, MMP9, and MMP13 in RA-FLS was detected by western blot and QRT-PCR assay. (B) The protein and mRNA expression of Rac1, Cdc42, and RhoA in RA-FLS was detected by western blot and qRT-PCR assay. ${ }^{*} p<0.05$, versus control mimic group. $\# p<0.05$, versus control inhibitor group. a candidate target miRNA of FSTL1, significantly decreased expression of miR-27a was shown in the serum, synovial tissue, and FLS of RA patients, compared to healthy controls (Fig. 1B).
These data suggest that a decrease in miR-27a expression and an increase in FSTL1 expression may be involved in the devel opment of RA. 
A

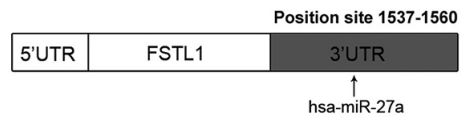

WT FSTL1 3'UTR 5'...auGGAGAUUUAAUCCUAAAACUGUGAC...3'

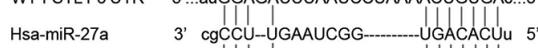
Mut FSTL1 3'UTR $\quad 5$ '...auĆCÚGUUUUAAUCCUAAAUUGÁCÁCÚc....3'

\section{B}

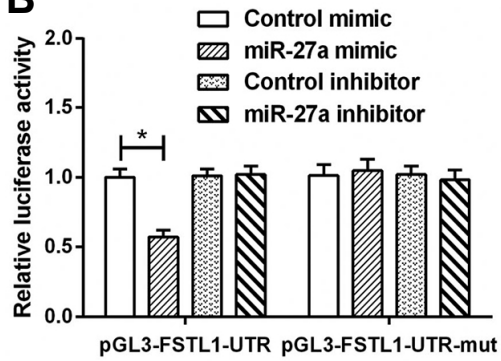

C
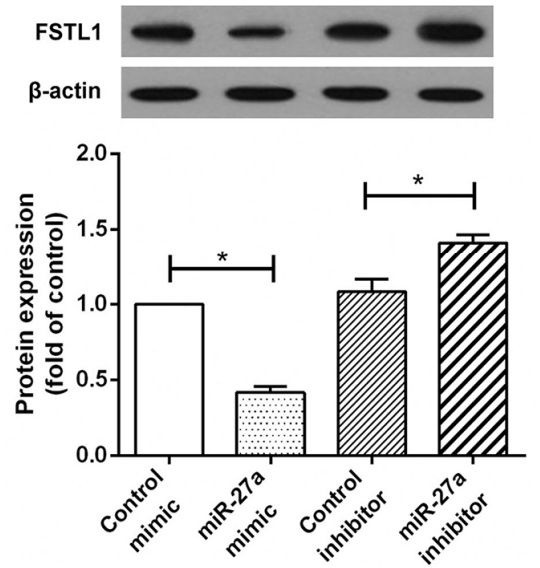

Fig. 4. Identification of FSTL1 as a target of miR-27a. (A) A luciferase reporter with either the wild-type or mutant sequences of FSTL1 3'UTR was constructed according to the sequence of the miR-27a binding site within the $3^{\prime}$ UTR of human FSTL1, using the pGL3 vector. (B) For the luciferase reporter assay, FLS were co-transfected with the luciferase reporter vectors and control mimic, miR-27a mimic, control inhibitor, or miR-27a inhibitor. Relative luciferase activity in RA-FLS was measured after a 48-h transfection. (C) Western blot analysis was used to evaluate the expression of FSTL1 in RA-FLS after transfection with miR-27a mimic or miR-27a inhibitor. ${ }^{*} p<0.01$ versus control group.

B
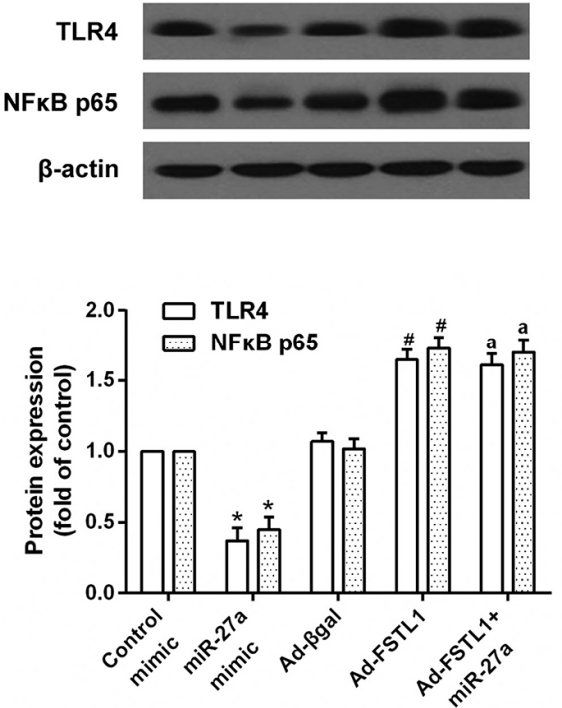

Fig. 5. FSTL1 overexpression rescues the miR-27a-mediated suppressive effect on RA-FLS migration and invasion by activating the TLR4/NFKB pathway. RAFLS were transfected with miR-27a, exogenous FSTL1, or miR-27a and exogenous FSTL1. (A) The cell migration and invasion of RA-FLS as measured using the Transwell system. (B) Protein expression levels of TLR4 and NFKB in RA-FLS were detected by western blot analysis. ${ }^{*} p<0.05$, versus control mimic group. ${ }^{*} p<0.05$, versus Ad- $\beta$-gal group. ${ }^{a} p<0.05$, versus miR27a mimic group.

\section{MiR-27a overexpression reduces cell migration and} invasion of RA FLS

Cell migration and invasion were detected in RA-FLS after the transfection of the miR-27a mimic or miR-27a inhibitor. The expression of miR-27a was significantly increased by transfection with miR-27a but significantly reduced by transfection with the miR-27a inhibitor (Fig. 2A), suggesting that the transfection efficiency was sufficient for further analysis. The results of the Transwell assay showed that the miR-27a mimic significantly inhibited cell migration and invasion of RA-FLS, whereas the miR-27a inhibitor promoted FLS migration and invasion in RA (Figs. 2B and 2C). These data suggest that miR-27a inhibits cell migration and invasion of RA-FLS.

\section{MiR-27a overexpression inhibits the expression of} migration and invasion-related proteins in RA-FLS To further determine the role of miR-27a in cell migration and invasion, the expression of migration and invasion-related proteins was detected by using western blot and qRT-PCR. It was shown that the expression of MMP2, MMP9, and MMP13 proteins was reduced by miR-27a, Whereas their expression was upregulated by miR-27a inhibitor. The qRT-PCR results indicated that MMP2, MMP9, and MMP13 mRNA levels were downregulated by miR-27a, as observed for protein levels in transfected FLS (Figs. 3A and 3B). In addition, miR-27a reduced the expression of Rac1, Cdc42, RhoA protein, and mRNA, which was upregulated by the miR-27a inhibitor (Figs. $3 \mathrm{C}$ and $3 \mathrm{D}$ ). These findings imply that miR-27a inhibits the expression of migration and invasion-related proteins in RA-FLS.

\section{MiR-27a directly targets FSTL1 expression}

In our study, FSTL1 was identified as a potential miR-27a target gene. To verify the binding site, the $3^{\prime}$-UTR of FSTL1 containing the wild type or mutated seed-sequence of miR-27a was 
cloned for use in a firefly luciferase reporter assay (Fig. 4A). Compared with the control and the miR-27a inhibitor, miR-27a significantly inhibited relative luciferase activity when cotransfected with the FSTL1-UTR reporter plasmid. However, miR-27a-mediated inhibitory effects were not observed in the mutant reporter transfected cells (Fig. 4B). Western blot results showed that the protein expression of FSTL1 was decreased in miR-27a-transfected RA FLS and increased in miR-27a inhibitor-transfected RA FLS (Fig. 4C). These results suggest that miR-27a may suppress FSTL1 expression by directly binding to the $3^{\prime}$-UTR of FSTL1.

FSTL1 overexpression rescues the miR-27a-mediated suppressive effect on RA-FLS migration and invasion by activating the TLR4/NFKB pathway

Previous studies show that miR-27a attenuated inflammatory damage by negatively regulating the TLR4/NFKB pathway (Li et al., 2015c; Lv et al., 2016). Thus, to evaluate the role of TLR4/NFKB signaling in RA-FLS migration and invasion, we examined the cell migration and invasion of RA-FLS, as well as the levels of TLR4 and NFKB in RA-FLS, which had been transfected with miR-27a and exogenous FSTL1. The results described herein showed that miR-27a reduced cell migration and invasion of RA-FLS, whereas these effects were restored by the eukaryotic expression and vector-mediated delivery of FSTL1 (Fig. 5A). Furthermore, miR-27a downregulated the mRNA and protein expression of TLR4 and NFKB, but overexpression of FSTL1 significantly counteracted this inhibitory effect (Fig. 5B). Taken together, these data indicate that overexpression of FSTL1 abrogates the reduction in miR-27areduced TLR4/NFKB activation and suggest that FSTL1 is a functional mediator of the effects of miR-27a in RA-FLS.

\section{DISCUSSION}

$\mathrm{RA}$ is an autoimmune disease correlating with the pathological features of joint synovial inflammation. FSTL1 is widely considered to participate in the development and pathogenesis immune diseases as a secreted extracellular glycoprotein (Chaly et al., 2012). MiRNA is a set of noncoding small RNA molecules found in recent years ( $\mathrm{Li}$ et al., 2010) that can regulate posttranscriptional mRNA expression and a variety of immune cell activation and differentiation processes (Duroux-Richard et al., 2011). It has been reported that miRNAs affect the proliferation and invasion of RA-FLS (Yang and Yang, 2015). Notably, miR27a was dysregulated in the chondrocytes of osteoarthritis (Tardif et al., 2009). However, the role of miR-27a in RA remains unclear. Our preliminary data showed that the expression of miR-27a, a candidate target miRNA of FSTL1, was significantly decreased in the serum, synovial tissue, and FLS of RA patients, compared with healthy controls. Considering the downregulation of miR-27a in RA patients, it may have a critical role in RA development. Thus, we investigated whether miR27a could suppress the migration and invasion of RA-FLS and migration-related protein expression by targeting FSTL1.

RA-FLS is the major cell population involved in the development of RA in synovial tissues. Previous studies have shown that RA-FLS migrate and invade the cartilage and bone, contributing to pannus formation and tissue damage during $R A$ progression (Lefevre et al., 2009). Therefore, modulation of RAFLS migration and invasion may offer a novel strategy for RA therapy (Li et al., 2015a). Prior studies indicated that the suppression of FLS migration and invasion might protect against joint destruction in RA (Lao et al., 2016). It has been demon- strated that miR-27a mimics impaired the invasive ability of human malignant glioma cell lines (Chen et al., 2015; Li et al., 2015b). Our study suggested that miR-27a inhibited the cell migration and invasion of RA-FLS. However, the inhibition of miR-27a suppressed the migration and invasion of breast cancer cells (Zhang et al., 2016). Thus, the effects of miR-27a on cell migration and invasion may vary in different tissues.

Previous studies have shown that MMPs produced by RAFLS are involved in the development and progression of RA (Yoshihara and Yamada, 2007). MMP13 promotes bone destruction in RA (Zhao et al., 2014). In addition, endogenous MMP2 and MMP9 contribute to synovial fibroblast survival, proliferation, migration, and invasion in RA (Pratheeshkumar and Kuttan, 2011). Increased activation of RhoA was found in ex vivo FLS from RA patients, as compared with those of healthy controls. The inhibition of RhoA activity could suppress the cytoskeletal reorganization and proliferation of RA-FLS. As the most well-known Rho family member, Rac1 has been reported to inhibit RA-FLS proliferation and invasion (Liang et al., 2013). Previous studies have indicated that downregulation of RhoA and Cdc42 inhibits the invasion and migration of osteosarcoma cells (Fukuda et al., 2016). The present study showed that miR-27a suppressed expression of MMPs and Rho family members and inhibited RA-FLS migration and invasion. These findings indicate that the attenuation of FLS migration and invasion induced by miR-27a may be achieved by inhibiting MMP and Rho family protein expression.

To further investigate the role of miR-27a in RA-FLS migration and invasion, RA-FLS were transfected with miR-27a and exogenous FSTL1. The results showed that miR-27a reduced the migration and invasion of RA-FLS, whereas the effects were restored by FSTL1 overexpression. There is increasing evidence that toll-like receptors play a key role in the development of RA in response to molecules of microbial origin found in the joints of RA patients. It has been demonstrated that FSTL1 can bind to CD14 via TLR4 (Murakami et al., 2012). Toll-like receptor signaling pathways culminate with activation of transcription factor NFKB and of mitogen-activated protein kinases JNK and p38. The control of toll-like receptor expression occurs at various levels, including post-transcriptionally; miRNAs have emerged as a new class of toll-like receptor regulators. TLR4 mRNA regulation by members of the let-7 miRNA family has been reported (Androulidaki et al., 2009). A previous study showed that miR-27a attenuates inflammatory damage by negatively regulating the TLR4/NFKB pathway ( $\mathrm{Li}$ et al., 2015c). The elevated level of serum FSTL1 suggests that the molecule is a potential serum marker related to the severity of joint damage in RA, perhaps by promoting RA-FLS migration and invasion. Our results showed that the TLR4/NFKB pathway was inhibited by miR-27a; the effects of miR-27a also were inhibited by FSTL1 overexpression. These data suggest that FSTL1 is a functional mediator of the effects of miR-27a in RAFLS. Moreover, considering the miR-27a downregulation and FSTL1 upregulation observed in RA patients, we propose that FSTL1 is a target gene of miR-27a in RA-FLS. Our findings suggest that miR-27a regulates the expression of FSTL1 in both mRNA and protein levels by directly binding to the $3^{\prime}$-UTR of FSTL1.

In conclusion, we have found that the novel microRNA miR$27 \mathrm{~b}$ functions as a RA-FLS migration and invasion suppressor. This suppression is associated with downregulation of MMPs and Rho family proteins in RA-FLS cells. In addition, the effects of miR-27b may depend on targeting FSTL1 with the inactivation of the TLR-4/NFKB pathway. Thus, our study provides evi- 
dence showing that miR-27a may be a useful target for the management of RA.

\section{REFERENCES}

Abeles, A.M., and Pillinger, M.H. (2006). The role of the synovial fibroblast in rheumatoid arthritis: cartilage destruction and the regulation of matrix metalloproteinases. Bull. NYU Hosp. Jt. Dis. 64, 20-24.

Aletaha, D., Neogi, T., Silman, A.J., Funovits, J., Felson, D.T., Bingham, C.O., 3rd, Birnbaum, N.S., Burmester, G.R., Bykerk, V.P., Cohen, M.D., et al. (2010). 2010 Rheumatoid arthritis classification criteria: an American College of Rheumatology/ European League Against Rheumatism collaborative initiative. Arthritis Rheum. 62, 2569-2581.

Androulidaki, A., lliopoulos, D., Arranz, A., Doxaki, C., Schworer, S., Zacharioudaki, V., Margioris, A.N., Tsichlis, P.N., and Tsatsanis, C. (2009). The kinase Akt1 controls macrophage response to lipopolysaccharide by regulating microRNAs. Immunity 31, 220231.

Bottini, N., and Firestein, G.S. (2013). Duality of fibroblast-like synoviocytes in RA: passive responders and imprinted aggressors. Nat. Rev. Rheumatol. 9, 24-33.

Chaly, Y., Marinov, A.D., Oxburgh, L., Bushnell, D.S., and Hirsch, R. (2012). FSTL1 promotes arthritis in mice by enhancing inflammatory cytokine/chemokine expression. Arthritis Rheum. 64, 1082-1088.

Chen, W., Qi, J., Bao, G., Wang, T., Du, C.W., and Wang, M.D. (2015). Emerging role of microRNA-27a in human malignant glioma cell survival via targeting of prohibitin. Mol. Med. Rep. 12, 1515-1523.

Clutter, S.D., Wilson, D.C., Marinov, A.D., and Hirsch, R. (2009). Follistatin-like protein 1 promotes arthritis by up-regulating IFNgamma. J. Immunol. 182, 234-239.

Ding, Q.H., Ji, X.W., Cheng, Y., Yu, Y.Q., Qi, Y.Y., and Wang, X.H. (2013). Inhibition of matrix metalloproteinases and inducible nitric oxide synthase by andrographolide in human osteoarthritic chondrocytes. Mod. Rheumatol. 23, 1124-1132.

Duroux-Richard, I., Presumey, J., Courties, G., Gay, S., Gordeladze, J., Jorgensen, C., Kyburz, D., and Apparailly, F. (2011) MicroRNAs as new player in rheumatoid arthritis. Joint Bone Spine 78, 17-22.

Edris, B. (2011). A comparison of the Oligomap and TargetScan algorithms for miRNA target analysis. Bmi231.stanford.edu.

Fassbender, H.G., and Simmling-Annefeld, M. (1983). The potential aggressiveness of synovial tissue in rheumatoid arthritis. J. Pathol. 139, 399-406.

Fukuda, H., Nakamura, S., Chisaki, Y., Takada, T., Toda, Y., Murata, H., Itoh, K., Yano, Y., Takata, K., and Ashihara, E. (2016). Daphnetin inhibits invasion and migration of LM8 murine osteosarcoma cells by decreasing RhoA and Cdc42 expression. Biochem. Biophys. Res. Commun. 471, 63-67.

Gallo, A., Tandon, M., Illei, G., and Alevizos, I. (2014). Discovery and validation of novel microRNAs in Sjogren's syndrome salivary glands. Clin. Exp. Rheumatol. 32, 761-762.

Karouzakis, E., Gay, R.E., Gay, S., and Neidhart, M. (2009) Epigenetic control in rheumatoid arthritis synovial fibroblasts. Nat. Rev. Rheumatol. 5, 266-272.

Lao, M., Shi, M., Zou, Y., Huang, M., Ye, Y., Qiu, Q., Xiao, Y., Zeng, S., Liang, L., Yang, X., et al. (2016). Protein inhibitor of activated STAT3 regulates migration, invasion, and activation of fibroblastlike synoviocytes in rheumatoid arthritis. J. Immunol. 196, 596606.

Lefevre, S., Knedla, A., Tennie, C., Kampmann, A., Wunrau, C., Dinser, R., Korb, A., Schnaker, E. M., Tarner, I. H., Robbins, P.D., et al. (2009). Synovial fibroblasts spread rheumatoid arthritis to unaffected joints. Nat. Med. 15, 1414-1420.

Li, J., Wan, Y., Guo, Q., Zou, L., Zhang, J., Fang, Y., Fu, X., Liu, H., Lu, L., and $\mathrm{Wu}, \mathrm{Y}$. (2010). Altered microRNA expression profile with miR-146a upregulation in CD4+ T cells from patients with rheumatoid arthritis. Arthritis Res. Ther. 12, R81.

Li, G.F., Qin, Y.H., and Du, P.Q. (2015a). Andrographolide inhibits the migration, invasion and matrix metalloproteinase expression of rheumatoid arthritis fibroblast-like synoviocytes via inhibition of HIF-1alpha signaling. Life Sci. 136, 67-72.
Li, W.Q., Yu, H.Y., Zhong, N.Z., Hou, L.J., Li, Y.M., He, J., Liu, H.M., Xia, C.Y., and Lu, Y.C. (2015b). miR27a suppresses the clonogenic growth and migration of human glioblastoma multiforme cells by targeting BTG2. Int. J. Oncol. 46, 1601-1608.

Li, X.Q., Lv, H.W., Wang, Z.L., Tan, W.F., Fang, B., and Ma, H. (2015c). MiR-27a ameliorates inflammatory damage to the blood-spinal cord barrier after spinal cord ischemia: reperfusion injury in rats by downregulating TICAM-2 of the TLR4 signaling pathway. J. Neuroinflammation 12, 25

Liang, L.Q., Huang, M.C., Qiu, Q., Xiao, Y.J., Sun, M.Y., Zhan, Z.P. Ye, Y.J., Fan, J.J., Yang, X.Y., and Xu, H.S. (2013) [Modulation of RhoA/Rho kinase on migration, invasion and proliferation of fibroblast like synoviocytes from patients with rheumatoid arthritis] Zhonghua Yi Xue Za Zhi 93, 1345-1348.

Liang, L., Huang, M., Xiao, Y., Zen, S., Lao, M., Zou, Y., Shi, M., Yang, X., and Xu, H. (2015). Inhibitory effects of niclosamide on inflammation and migration of fibroblast-like synoviocytes from patients with rheumatoid arthritis. Inflamm Res. 64, 225-233.

Luo, X., Tsai, L.M., Shen, N., and Yu, D. (2010). Evidence for microRNA-mediated regulation in rheumatic diseases. Ann. Rheum. Dis. 69 Suppl 1, i30-36.

Lv, Q., Zhu, X.Y., Xia, Y.F., Dai, Y., and Wei, Z.F. (2015). Tetrandrine inhibits migration and invasion of rheumatoid arthritis fibroblastlike synoviocytes through down-regulating the expressions of Rac1, Cdc42, and RhoA GTPases and activation of the PI3K/Akt and JNK signaling pathways. Chin J. Nat. Med. 13, 831-841.

Lv, Y.N., Ou-Yang, A.J., and Fu, L.S. (2016). MicroRNA-27a negatively modulates the inflammatory response in lipopolysaccharidestimulated microglia by targeting TLR4 and IRAK4. Cell Mol. Neurobiol. [Epub ahead of print]

McInnes, I.B., and Schett, G. (2011). The pathogenesis of rheumatoid arthritis. N Engl. J. Med. 365, 2205-2219.

Murakami, K., Tanaka, M., Usui, T., Kawabata, D., Shiomi, A., Iguchi-Hashimoto, M., Shimizu, M., Yukawa, N., Yoshifuji, H., Nojima, T., et al. (2012). Follistatin-related protein/follistatin-like 1 evokes an innate immune response via CD14 and toll-like receptor 4. FEBS Lett. 586, 319-324.

Nakasa, T., Shibuya, H., Nagata, Y., Niimoto, T., and Ochi, M. (2011). The inhibitory effect of microRNA-146a expression on bone destruction in collagen-induced arthritis. Arthritis Rheum. 63 , 1582-1590.

O'Connell, R.M., Rao, D.S., and Baltimore, D. (2012). MicroRNA regulation of inflammatory responses. Annu. Rev. Immunol. 30, 295-312.

Okamoto, H., Shidara, K., Hoshi, D., and Kamatani, N. (2007). Antiarthritis effects of vitamin $\mathrm{K}(2)$ (menaquinone-4)--a new potential therapeutic strategy for rheumatoid arthritis. FEBS J. 274, 45884594.

Pan, W., Wang, H., Jianwei, R., and Ye, Z. (2014). MicroRNA-27a promotes proliferation, migration and invasion by targeting MAP2K4 in human osteosarcoma cells. Cell Physiol. Biochem. 33, 402-412.

Pratheeshkumar, P., and Kuttan, G. (2011). Andrographolide inhibits human umbilical vein endothelial cell invasion and migration by regulating MMP-2 and MMP-9 during angiogenesis. J. Environ. Pathol. Toxicol. Oncol. 30, 33-41.

Shibanuma, M., Mashimo, J., Mita, A., Kuroki, T., and Nose, K. (1993). Cloning from a mouse osteoblastic cell line of a set of transforming-growth-factor-beta 1-regulated genes, one of which seems to encode a follistatin-related polypeptide. Eur. J. Biochem 217, 13-19.

Tardif, G., Hum, D., Pelletier, J.P., Duval, N., and Martel-Pelletier, J. (2009). Regulation of the IGFBP-5 and MMP-13 genes by the microRNAs miR-140 and miR-27a in human osteoarthritic chondrocytes. BMC Musculoskelet Disord. 10, 148.

Yang, S., and Yang, Y. (2015) Downregulation of microRNA221 decreases migration and invasion in fibroblastlike synoviocytes in rheumatoid arthritis. Mol. Med. Rep. 12, 2395-2401.

Yoshihara, Y., and Yamada, H. (2007). Matrix metalloproteinases and cartilage matrix degradation in rheumatoid arthritis. Clin. Calcium 17, 500-508

Yuan, H., Yang, P., Zhou, D., Gao, W., Quu, Z., Fang, F., Ding, S. and Xiao, W. (2014). Knockdown of sphingosine kinase 1 inhibits the migration and invasion of human rheumatoid arthritis fibroblast-like synoviocytes by down-regulating the PI3KJAKT activation and MMP-2/9 production in vitro. Mol. Biol. Rep. 41, 
miR-27a Inhibits Migration and Invasion of FLSs

Dong-liang Shi et al.

5157-5165.

Zhang, X.H., Xu, X.X., and Xu, T. (2015). Ginsenoside Ro suppresses interleukin-1 beta-induced apoptosis and inflammation in rat chondrocytes by inhibiting NF-kappaB. Chin J. Nat. Med. 13, 283-289.

Zhang, S., Ma, C., Pang, H., Zeng, F., Cheng, L., Fang, B., Ma, J., Shi, Y., Hong, H., Chen, J., et al. (2016). Arsenic trioxide suppresses cell growth and migration via inhibition of miR-27a in breast cancer cells. Biochem. Biophys. Res. Commun. 469, 5561.

Zhao, W., Zhang, C., Shi, M., Zhang, J., Li, M., Xue, X., Zhang, Z. Shu, Z., Zhu, J., Mu, N., et al. (2014). The discoidin domain receptor 2/annexin A2/matrix metalloproteinase 13 loop promotes joint destruction in arthritis through promoting migration and invasion of fibroblast-like synoviocytes. Arthritis Rheumatol. 66, 2355-2367. 